\title{
Abnormal thyroid test results in euthyroid state: An appraisal of the role of drugs
}

\author{
Aishwarya Krishnamurthy ${ }^{1}$, Saptarshi Bhattacharya ${ }^{1}$, Tejal Lathia ${ }^{2}$, and Nilakshi Deka ${ }^{3}$ \\ ${ }^{1}$ Max Balaji Hospital Patparganj \\ ${ }^{2}$ Fortis Hospital, Vashi \\ ${ }^{3}$ Apollo Hospitals Guwahati
}

July 7, 2020

\begin{abstract}
Background: Thyroid function tests (TFTs) are one of the most frequently performed hormonal tests. Abnormal reports are commonly encountered even in the absence of thyroid dysfunction with medication use during testing being an important cause of this anomaly. Knowledge about the effects of different drugs on various components of TFTs will prevent unnecessary referrals, investigation and potentially dangerous therapeutic decisions. Objective: This narrative review aims to provide information to physicians regarding how various medicines can alter results of TFT even in the euthyroid state. Methods: Articles published up to April, 2020 in English language were identified through searches in PubMed and Google Scholar with a search strategy using keywords appropriate to analyze the effects of different medications on thyroid assays. Results: Medications ranging from over-the-counter vitamins to anti-cancer drugs are associated with anomalous TFTs in euthyroid individuals. This occurs through mechanisms of assay interference and alterations in levels or affinity of thyroid binding proteins (TBPs). High-dose biotin can mimic findings seen in thyrotoxicosis by interference with immunoassays utilizing streptavidin-biotin immobilizing systems. Heparin-induced increase in non-essential fatty acid causes displacement of thyroxine (T4) from TBP, effecting an artefactual increase in free hormone levels. Increases or decreases in thyroid-binding globulin (TBG), the key TBG, can cause a corresponding change in total T4 and tri-iodothyronine levels. Estrogen, selective estrogen receptor modulators, 5-fluorouracil, mitotane, clofibrate, heroin and methadone can increase while androgens, anabolic steroids, glucocorticoids, nicotinic acid and l-asparaginase can decrease TBG. Salicylates, frusemide, carbamazepine and phenytoin may displace T4 from TBP, producing a decline in the total hormone levels. Conclusions: Many categories of medications can interfere with analysis of TFTs through different mechanisms. Awareness about these drug-induced TFT fallacies is essential to avoid improper diagnosis and overtreatment of euthyroid patients.
\end{abstract}

\section{Review criteria:}

The information was collected by a search of the English language literature in PubMed and Google Scholar from inception up to April, 2020. Relevant search words, pertaining to drugs and thyroid assays were used. References obtained from original papers were also scrutinized and included wherever relevant to the subject of the review.

\section{Message for the clinic:}

The current manuscript will sensitise readers to the effects of drugs on thyroid assays. Abnormal TFTs should always be correlated with the clinical profile of the patient. The importance of eliciting a meticulous medication history, in preventing unnecessary investigations or inappropriate therapeutic decisions, cannot be over-emphasized.

\section{Introduction}


Thyroid function test (TFT) is one of the most commonly performed hormonal evaluations. Abnormalities can be detected in TFT reports in euthyroid individuals due to various reasons. One such important, but overlooked cause is the interference from concomitant medication usage. Several drugs, ranging from over-the-counter (OTC) vitamin supplements to specialised anti-cancer medicines can affect the estimation of any of the components of TFT [thyroid-stimulating hormone (TSH), total thyroxine (TT4), total triiodothyronine (TT3), free thyroxine (FT4) or free tri-iodothyronine (FT3)].

This review will focus on those drugs that produce fallacious TFTs, in the absence of any clinically relevant effect on thyroid hormone secretion. Many of the medications actually affect thyroid functioning and cause hypothyroidism, thyrotoxicosis, thyroiditis, etc. Those drugs have not been discussed in this review. Awareness about these drug-induced TFT alterations could avert misdiagnosis and overtreatment of euthyroid subjects.

\section{Methodology}

We searched PubMed and Google Scholar for eligible publications and articles published before April, 2020 using the following search strategy. We used one of the following terms: "thyroid function test", "thyroid assay", "thyroid stimulating hormone", "thyroxine", "tri-iodothyronine", "thyroid binding globulin", "thyroid binding proteins" in combination with "drugs", "medications", "assay interference", "biotin", "heparin", "oral contraceptive pills", "selective estrogen receptor modulators", "estrogen", "tamoxifen", "raloxifene", "droloxifene", "fluorouracil", "clofibrate", "heroin", "methadone", "mitotane", "nicotinic acid", "asparaginase", "glucocorticoids", "androgens", "anabolic steroids", "l-asparaginase", "salicylates", "aspirin", "salsalate", "non-steroidal anti-inflammatory drugs", "frusemide", "phenytoin" and "carbamazepine". References obtained from original papers were also scrutinized and included wherever relevant to the subject of this review.

\section{Drugs interfering with the assessment of thyroid function tests.}

Principal mechanisms by which drugs can give rise to spurious thyroid test results are as follows:

1. Assay interference

2. Changes in levels of serum thyroid binding proteins (TBPs) - particularly thyroid-binding globulin (TBG)

3. Alteration in affinity or binding of thyroid hormones to serum TBP.

The common medications which modify thyroid test results by the above processes shall be discussed in the following sections.

\section{Drugs causing assay interference}

Medications can interfere with the accurate estimation of the thyroid profile due to interference with components of the assays used. Biotin and heparin are commonly used medications that cause TFT abnormalities in euthyroid individuals by distinct mechanisms.

\section{Biotin}

Biotin based Immunoassays: Biotin is a water-soluble vitamin belonging to vitamin B family. It is also an essential component of streptavidin-biotin immobilising systems (SBIS) used for most immunoassays. This system exploits the interaction between the fungal protein streptavidin and biotin which is one of the strongest non-covalent interactions in nature. It remains undisturbed by multiple washing steps in assays. ${ }^{1}$ Furthermore, biotinylation does not alter the biological activity or immunologic specificity when bound to any test molecule. SBIS is widely used in many Food and Drug Administration approved immunoassay systems using fully automated platforms, including Access, DxI, and DxC (Beckman Coulter, California, US); the Elecsys, Cobas, and Modular platforms (Roche Diagnostics, Basel, Switzerland); the Isys platform (Immuno Diagnostic System, East Boldon, United Kingdom); the Ortho Vitros platform (Ortho Clinical Diagnostics, New Jersey, US); the Dimension Vista, Exl, Immulite platforms (Siemens Healthineers, Erlangen, 
Germany), Abbott Architect i2000@ (Abbott Diagnostics, Illinois, United States), and Diasorin Liaison XL@ (DiaSorin, Saluggia, Italy). ${ }^{2}$

Type of abnormality: The presence of excess biotin in test samples causes abnormalities in accordance with the type of immunoassay. Falsely low readings occur with sandwich immunoassays (glycoprotein hormones like TSH) and falsely high values in competitive immunoassays (e.g. triiodothyronine (T3), thyroxine (T4), steroid hormones and 25-hydroxy-vitamin D). ${ }^{2}$

1. Mechanism of interference with sandwich immunoassays: When the assay has a "sandwich" design as employed for estimation of TSH, the test serum is incubated with biotinylated monoclonal TSH antibodies and radiolabelled monoclonal antibodies. Immune complex "sandwiches" thus formed are captured by streptavidin-coated magnetic microparticles. Chemiluminescence produced by application of a voltage to these magnetic microparticles is directly proportional to the TSH levels in the test serum. The presence of excess biotin in the serum will saturate the streptavidin binding sites and reduce binding of these immune sandwiches to the solid phase (magnetic microparticles) resulting in low chemiluminescence and falsely low readings as illustrated in Figure 1.

2. Mechanism of interference with competitive immunoassays: When a competitive immunoassay is utilised as in the $\mathrm{FT}_{4}$ assay, the test serum is incubated with biotinylated $\mathrm{T} 4$ molecules and radiolabelled anti-T4 monoclonal antibodies. Biotinylated T4 molecules compete with the analyte (or T4) in the test serum for binding with the radiolabelled anti-T4 antibodies. The biotinylated T4 molecules are then immobilised in the streptavidin-coated microparticles. The chemiluminescence signal generated from the application of voltage to these magnetic microparticles is inversely proportional to the levels of $\mathrm{FT}_{4}$. The presence of excess biotin in the test serum will prevent the complexes of biotinylated T4-radiolabelled antibodies from binding to the magnetic microparticles. This would result in lower chemiluminescence, translating to falsely higher levels of T4 in the serum as depicted in Figure 2.

Biotin dose and assay interference: The recommended daily intake of biotin ranges from 30-70 mcg daily. While dietary biotin intake does not generate significant enough blood levels to interfere with in-vitro diagnostic tests, supraphysiologic levels achieved through the therapeutic use of biotin as supplements for skin and hair growth, multiple sclerosis and rare inherited metabolic disorders like biotinidase deficiency, propionic academia, thiamine responsive basal ganglia disease, holocarboxylase synthase deficiency and mitochondrial disorders, can cause problems with these tests. ${ }^{3}$ The minimal dose required and the degree, duration, and magnitude of this interference is variable and might be specific to the analyte being tested and the assay characteristics. ${ }^{4,5}$ The level of interference depends on the serum concentration attained rather than the dose of biotin consumed, which in turn is determined by the length of the washout period before testing. ${ }^{6,7}$ Grimsey et al. evaluated washout periods, required for assays with interference thresholds ranging from 10 to $100 \mathrm{ng} / \mathrm{ml}$, at biotin dose regimens ranging from $1 \mathrm{mg}$ once daily to $300 \mathrm{mg}$ four times daily. ${ }^{7}$ For assays with an in-vitro interference threshold of more than $30 \mathrm{ng} / \mathrm{ml}$, biotin doses of up to $5 \mathrm{mg}$ twice daily or 10 mg once daily, an 8-hr washout period is sufficient to mitigate the risk. If assays have an in-vitro interference threshold of $<30 \mathrm{ng} / \mathrm{ml}$, or in rare cases of biotin intake of more than $10 \mathrm{mg}$ per day, sampling should be delayed for a more extended period (up to $73 \mathrm{~h}$ ) after the last dose of biotin. Though the effect of biotin on FT3, FT4 and TSH estimation wanes in hours, anti-TSH receptor antibodies (TRAbs), which can also be falsely elevated due to this assay interference, may take up to 7 days to normalise. ${ }^{2,8-10}$

Clinical correlate: The TFT could falsely suggest a diagnosis of overt or subclinical thyrotoxicosis or thyroid hormone resistance, and falsely elevated TRAbs can further mislead the diagnosis. History of intake of OTC vitamin supplements containing biotin should be elicited. If an erroneous report is suspected, retesting should be done in serial dilutions (if using the same platform) or on another platform which does not utilise SBIS (Centaur FT4, Diasorin, Abbott). ${ }^{11,12}$ Another option would be to repeat the test, after stopping biotin supplements for a duration which is determined by the dosage and analyte being assessed. Depletion protocols which involve pre-treatment of the test sample with substances (e.g. streptavidin-coated particles) that bind biotin can also be used. ${ }^{13}$

\section{Heparin}


TFTs performed in individuals receiving heparin, can demonstrate fallaciously elevated FT4 and FT3, with normal TSH levels. ${ }^{14,15}$

Mechanism of interference: Heparin induces release of lipoprotein lipase (LPL) from vascular endothelium, which acts on triglycerides, results in an increase in serum levels of non-esterified fatty acids (NEFA). High NEFA levels inhibit binding of thyroid hormone with TBP, producing an increase in the measured free hormone levels. ${ }^{14}$ There is a demonstrable increase in blood levels of LPL as well as FFA, that persists in-vitro, in patients receiving heparin. ${ }^{14,15}$

Relation to the type of heparin: This effect has been reported with the use of unfractionated heparin (UFH) as well as low molecular weight heparin (LMWH), irrespective of the route or dose. Standard subcutaneous doses, as well as intravenous doses as small as $0.08 \mathrm{U} / \mathrm{kg}$, can cause a significant increase in LPL activity of the serum. ${ }^{16}$ It takes ten hours or more after UFH injection for this effect to remit. ${ }^{17}$ Greater bioavailability and longer duration of action of LMWH might necessitate an interval of $24 \mathrm{hrs}$ after the last dose for this effect to wane.

Variations depending on assay and storage: This interference has been observed with different assays, including direct immunoassays, ultracentrifugation, and equilibrium dialysis. ${ }^{18}$ Assays that require longer incubation periods (e.g. equilibrium dialysis) show maximum derangement. As an extension of the same effect, preanalytical delays due to storage of samples before testing can also worsen this interference as in-vitro displacement of FT4 and FT3 continues. $^{14}$

Physiologic variations: These TFT abnormalities are not seen in all patients receiving heparin as there are other factors at play, namely serum levels of triglycerides and albumin. The released LPL requires an adequate concentration of the substrate i.e. triglycerides $(>180 \mathrm{mg} / \mathrm{dl})$ in the serum to cause an increase in FFA. Albumin acts as a high-affinity binding protein for FFA; thus its serum concentration affects the FFA-induced effects on thyroid hormone displacement. FT4 levels remain unaltered, till such time that the molar concentration of FFA is five times more than that of albumin, overwhelming its binding capacity. ${ }^{19}$

Clinical correlate: Collecting blood samples after an adequate gap (at least 10 hours with UFH), immediate processing and testing and correlation with TSH can mitigate the problem. Estimating TT4 instead of FT4 in individuals receiving heparin specially in those having hypertriglyceridemia can be helpful. ${ }^{20}$

\section{Drugs affecting thyroxine-binding globulin}

\section{Thyroxine- binding globulin physiology}

TBG is a high affinity, low concentration TBP that binds $80 \%$ of T3 and $75 \%$ of T4. Transthyretin (TTR) and serum albumin are the other TBPs. TBG has a distributive as well as buffering role to stabilise free thyroid hormone concentration supply to target tissues. ${ }^{22}$ Abnormalities in the TBG can be inherited or acquired, and drugs remain a common cause of acquired alteration.

\section{Increase in thyroxine-binding globulin}

Estrogen: An elevated TT4 and TT3 level can be seen in patients receiving estrogen, while FT4, FT3 and TSH values remain normal. It causes a dose-dependent increase in TBG, commencing in two weeks of initiation and reaching a new steady-state within four to eight weeks. At routine doses of ethinyl estradiol (20-35 mcg) or conjugated equine estrogen (CEE) $(0.625 \mathrm{mg})$, there is an approximate $30-50 \%$ increase in TBG and $20-35 \%$ in TT4. ${ }^{23-27}$ The progesterone component of oral contraceptives (OCs) per se does not alter this estrogen-induced effect; however, the androgenic properties of the progesterone do offset the net effect of the OC on TBG levels. OCs containing anti-androgenic progesterone like dienogest induce a higher rise in TBG (50-60\% vs 30\%) as compared to those containing androgenic levonorgestrel. ${ }^{28}$ Transdermal estrogens do not affect TBG levels despite achieving comparable serum levels to oral formulations suggesting the possibility of a portal threshold for estrogenic stimulation of TBG. ${ }^{24,29}$

Estrogen increases TBG levels predominantly by prolonging its half-life, along with a possible increase in synthesis. It induces a post-translational modification of TBG, resulting in molecules which are more resistant 
to hepatic degradation, due to the higher terminal sialic acid content. ${ }^{30}$ The stimulatory effect of estrogen on TBG synthesis, has been observed in in-vitro studies. ${ }^{31,32}$

Selective estrogen receptor modulators: Selective estrogen receptor modulators (SERMs) are molecules that can act as agonist or antagonist at the estrogen receptors, depending on the target tissue.

1. Tamoxifen: Tamoxifen has a weak hepatic estrogen-agonistic effect and can cause a mild increase in serum TBG concentrations. The increases are lower than those observed during pregnancy or with estrogen use. ${ }^{33-36}$

2. Raloxifene : Raloxifene at a daily dose of $60 \mathrm{mg}$ is used for postmenopausal osteoporosis. Six months of therapy with raloxifene did not significantly affect TBG or TT4 levels. ${ }^{37}$ However when the same dose of raloxifene was administered for one year, it resulted in a small but significant increase in serum TBG. ${ }^{38,39}$

3. Droloxifene : In postmenopausal women, droloxifene at a dose of $60 \mathrm{mg}$ daily for six weeks, increased TBG by $41 \%$ from baseline. The increase in TBG with CEE (used as the comparator) was twice that observed with droloxifene. Although structurally similar to tamoxifen, droloxifene may have a more profound effect on serum TBG. It is unclear whether this is due to greater estrogen agonistic properties on the liver or is dose-related. ${ }^{40}$ Heroin and methadone : Serum TBG concentrations are increased in about $25-50 \%$ of chronic heroin abusers or those under methadone therapy. ${ }^{41-43}$ In a large series of 285 euthyroid narcotic addicts, $22 \%$ had elevated TT4 and TBG, with normalisation of both parameters after successful stabilisation with methadone therapy. ${ }^{44}$ Inhalational opium use is also associated with an increase in TT3 levels and TBG as assessed by T3 resin uptake (T3RU). ${ }^{45,46}$ T3RU is commonly used to evaluate TBG as there is an inverse association between T3RU and TBG. ${ }^{47}$

The mechanisms involved in these changes have not been clearly understood. The rise in TBG could be related to increased synthesis or decreased degradation or both.

The widely accepted theory is that of concomitant liver dysfunction, as TBG is synthesised in the liver. ${ }^{41,44,48}$ In methadone treated addicts, high T3 binding ratio has been demonstrated to correlate with the degree of hepatic dysfunction directly. ${ }^{48}$ The hepatic dysfunction in these patients is multifactorial with contribution from the direct effect of opiates on the liver, dietary deficiencies, chronic and low-grade inflammation due to frequent injections, abuse of other drugs and OC use. ${ }^{48}$ Liver dysfunction, however, is unlikely to be the sole pathogenetic mechanism as these TBG changes are also seen in addicts (8-25\%) with normal liver enzymes. ${ }^{44}$

The other proposed mechanism is through inhibition of thyroxine-metabolising microsomal enzymes in the liver by morphine (a heroin metabolite), contaminants (like quinine) and methadone. ${ }^{41,42}$ Alteration in sex steroids by hepatic dysfunction or direct effects of heroin or methadone could also influence TBG levels. ${ }^{41}$

5-Fluorouracil: 5-Fluorouracil has been associated with an increase in TT4 and TT3, but FT4 and TSH remain unchanged. It is probably due to an increase in TBG levels. There are not many studies reporting this effect of 5 -fluorouracil. ${ }^{49}$

Mitotane: Long term use of mitotane is associated with an increase in the concentration of TBG and also a smaller increment of sex hormone-binding globulin and cortisol binding globulin. The changes occur as early as one month after commencement of therapy and normalise gradually approximately one year after cessation of medications. The exact mechanisms causing this change in the binding proteins has remained elusive, and presumed to be related to reduced degradation due to enhanced sialylation or increased synthesis. ${ }^{50}$

Interestingly, unlike the other medications which raise TBG, there is a decrease in TT4 levels by around $38 \%$ from the baseline value. ${ }^{50}$ This is hypothesised to be due to competition of mitotane for binding sites on TBG or due to alteration in T4 binding characteristics. ${ }^{51}$

There are also reports of reduced FT4 levels with prolonged mitotane use, with an inverse correlation between FT4 levels and serum mitotane concentrations. ${ }^{52,53}$ While the exact reason for this is unknown, in patients receiving mitotane, high FT3/FT4 ratios have been demonstrated which indicate augmented deiodinase 
activity aiding the conversion of $\mathrm{T} 4$ to $\mathrm{T} 3{ }^{52,53}$ This is considered as a characteristic, compensatory thyroid function change in hypothyroid conditions. ${ }^{54}$ Changes in FT3 or TSH levels have not been reported.

Clofibrate: Clofibrate, a hypolipidemic agent, has been discontinued since 2002, given the observed excess mortality despite successful cholesterol-lowering. ${ }^{55}$ Increase in TBG and a small decrease in FT4 and FT3 levels were observed with this medication. ${ }^{56}$ There was however no relationship between these changes and the lipid response to clofibrates. ${ }^{56,57}$

\section{Decrease in thyroid-binding globulin}

\section{Androgens and anabolic steroids:}

Androgens when used as replacement therapy in hypogonadal men or in supraphysiological doses for anabolic effects in athletes of both sexes, result in decreased TBG, TT4 and TT3 levels without affecting FT4 and TSH levels. ${ }^{58-61}$ The rise starts within one week, reaching a maximum in two to three weeks. ${ }^{60}$

This effect has also been observed with danazol used in its usual dose of $800 \mathrm{mg}$ daily for treatment of endometriosis. ${ }^{62}$ Androgen therapy with the non-aromatizable oral fluoxymesterone for metastatic, hormonesensitive breast cancer, decreased serum levels of TT4 and TBG in four weeks. Normalisation occurs within six to twelve weeks of therapy cessation. In hypothyroid women, on levothyroxine supplementation, the decrease in TBG caused clinical thyrotoxicosis (with elevated FT4 and suppressed TSH), requiring 25 to $50 \%$ dose reduction in thyroid hormone doses. This effect reversed after 8-10 weeks of cessation of androgen therapy. ${ }^{63}$ In euthyroid female-to-male transsexuals, administration of testosterone esters at a dose of $250 \mathrm{mg}$ intramuscular every two weeks, was associated with a 14\% decrease in TBG but serum FT4 and TSH levels remained unchanged. This study also reported an increase in T3/ T4 ratio indicating a probable upregulation of deiodinase activity by testosterone. ${ }^{29}$

Though the exact mechanism involved is unclear; but it is believed that androgens cause desialylation of TBG making it susceptible to degradation. ${ }^{63}$ The net effect of a particular androgen on TBG levels depends on its aromatisation rate to estrogen. ${ }^{60}$ Adequacy of liver function also influences this effect, as observed in a study in males with alcoholic cirrhosis. Oral testosterone treatment resulted in TBG decrease in men with alcoholic cirrhosis and mild liver dysfunction (Child-Turcotte's group A) and not in those with more advanced liver disease (Child-Turcotte's group B or C). This could be due to inadequate expression of sex hormone receptors in the liver, increase in serum estrogen concentration and use of antiandrogenic medication like spironolactone. ${ }^{64}$ Short term studies have suggested a possible shift of T4 binding from TBG to transthyretin during therapy with norethandrolone due to lower TBG levels. ${ }^{65}$ From a clinical perspective, androgen therapy may unmask or worsen mild hyperthyroidism due to reduction of TBG levels. ${ }^{66}$

Chronic glucocorticoid therapy: Endogenous glucocorticoid hypersecretion or exogenous glucocorticoid administration, at a rate of 2-20 times the basal adrenal glucocorticoid secretion, results in decreased TBG levels. ${ }^{67-69}$ While it can occur with any route of administration, it depends on the dose and duration of the glucocorticoid therapy ${ }^{69}$ It is unclear how glucocorticoids cause this change, it is probably mediated through a decrease in the hepatic synthesis of TBG perhaps at a transcriptional level. ${ }^{68}$

L-asparaginase: L-asparaginase has been reported to decrease TBG levels, with a return to normal after withdrawal. It is believed to be due to the dose-dependent suppression of hepatic synthesis of the glycoprotein as demonstrated in in-vitro studies of cultured human hepatoma (HEP G2) cell lines. ${ }^{70}$ During the induction phase of acute lymphoblastic leukemia (ALL) treatment, L-asparaginase causes a reduction in TT4 and TBG. ${ }^{71-73}$ It occurs within two days to two weeks after administration and resolves in two to four weeks after cessation of medication. Two cases of transient hyperthyroidism occurring after l-asparaginase therapy for ALL, have been reported. There was an increase in FT4 along with TSH suppression accompanied by clinical features of thyrotoxicosis in both the cases. ${ }^{74}$

The mechanism involved is presumed to be due to the general ability of l-asparaginase to inhibit protein synthesis in the liver including suppressing the production of TBG. ${ }^{70}$ The mechanism of the single reported observation of an increase in FT4 is unexplained. 
Nicotinic acid: Nicotinic acid can result in decreased TBG and TT4 levels without affecting FT4 and TSH values. ${ }^{75-78} \mathrm{O}$ 'Brien et al. proposed nicotinic acid associated hepatitis or subclinical liver dysfunction, while Drinka et al. attributed a direct drug effect as the cause for the change in TBG. ${ }^{76,77}$ Shakir et al. ascribed mobilisation of fatty acids from the periphery, with subsequent interference to binding of thyroid hormones to TBG as another mechanism. ${ }^{75}$ The exact mechanism is still not clearly understood.

\section{Drugs altering the binding of thyroid hormone to thyroid binding proteins}

Displacement of thyroid hormones from TBPs like TBG and TTR can alter thyroid test results.

Salicylates and non-steroidal anti-inflammatory drugs(NSAIDs):Salicylates and salsalate inhibit binding of T4 to all three TBP with maximum effect on TBG ${ }^{79,80}$ This occurs at doses of more than $2 \mathrm{~g}$ daily of salicylates and 1.5-3 g daily of salsalate. There is an initial and transient increase in FT4 levels at drug initiation, which is then followed by a decrease in TT4 and normalisation of FT4 levels as therapeutic concentrations are maintained. ${ }^{81}$ Salsalate may produce a more significant decline in T4 levels as compared to salicylate (30-40\% vs $20-30 \%) .80,82$

An analysis of different NSAIDs revealed that ibuprofen, naproxen and indomethacin did not alter hormone levels, aspirin caused a decrease in TT4, TT3, TSH after one week and salsalate affected a drop of , FT4 levels (probably due to dilutional artefact). Meclofenamate caused transient fluctuation of hormone levels which normalised in a week. All subjects remained clinically euthyroid during the one week study period, and TSH remained within normal range. ${ }^{83}$

Frusemide: Frusemide displaces thyroid hormones from TBG, TTR and albumin, causing a transient increase in FT4 and decrease in TT4. ${ }^{84}$ This effect occurs at large intravenous doses of more than $80 \mathrm{mg}$ and not at usual therapeutic doses. ${ }^{67,85}$ The changes depend on the interval between drug administration and sample collection, occurring two to five hours after frusemide doses of 80,120 or $250 \mathrm{mg} .{ }^{86}$ Other factors involved are the renal drug clearance rate and serum concentration of albumin, which also binds frusemide. ${ }^{67,85}$

Phenytoin and Carbamazepine: Phenytoin and carbamazepine have been shown to lower T4, T3, FT3 and FT4 levels without affecting serum TSH levels, in euthyroid individuals. ${ }^{86}$ These drugs at therapeutic levels displace thyroid hormones from thyroid-binding proteins. This is distinct from the class effect of augmented T4 and T3 metabolism, by induction of hepatic microsomal P-450 enzymes. Low FT4 levels are presumably artefactual, and is believed to occur due to serum dilution during the estimation process. ${ }^{87}$ Direct measurement of FT4 by equilibrium dialysis or indirect assessment by measuring FT4 index can overcome this inaccuracy. ${ }^{88}$

\section{Conclusions}

Several classes of medications can cause abnormal TFT results, in euthyroid subjects, without any clinically apparent effect on thyroid hormone secretion. The possible mechanisms are through assay interference or changes in TBP levels or alterations in their affinity for thyroid hormones. Awareness of these drug-related effects, is of paramount importance as many of these changes, while not warranting any medical intervention, can misguide the clinician in diagnostic and therapeutic decision making.

The authors would like to acknowledge Ms. Abhilasha Krishnamurthy, December Design Studio, New Delhi for designing the illustrations.

\section{References}

1. Diamandis EP, Christopoulos TK. The biotin-(strept)avidin system: principles and applications in biotechnology. Clin Chem . 1991;37(5):625-636.

2. Odhaib SA, Mansour AA, Haddad NS. How Biotin Induces Misleading Results in Thyroid Bioassays: Case Series. Cureus . Published online May 23, 2019. doi:10.7759/cureus.4727 
3. Evans N, Yates J, Tobin J, McGill J, Huynh T. Immunoassay interference secondary to therapeutic highdose biotin: A paediatric case report: Biotin interference and thyroid function tests. $J$ Paediatr Child Health . 2018;54(5):572-575. doi:10.1111/jpc. 13857

4. Sturgeon CM, Viljoen A. Analytical error and interference in immunoassay: minimizing risk. Ann Clin Biochem . 2011;48(Pt 5):418-432. doi:10.1258/acb.2011.011073

5. Al-Salameh A, Becquemont L, Brailly-Tabard S, Aubourg P, Chanson P. A Somewhat Bizarre Case of Graves Disease Due to Vitamin Treatment.J Endocr Soc . 2017;1(5):431-435. doi:10.1210/js.2017-00054

6. Paketçi A, Köse E, Gürsoy Çalan Ö, et al. Serum Level of Biotin Rather than the Daily Dosage Is the Main Determinant of Interference on Thyroid Function Assays. Horm Res Paediatr . 2019;92(2):92-98. doi: $10.1159 / 000502740$

7. Grimsey P, Frey N, Bendig G, et al. Population pharmacokinetics of exogenous biotin and the relationship between biotin serum levels and in vitro immunoassay interference. Int J Pharmacokinet . 2017;2(4):247-256. doi:10.4155/ipk-2017-0013

8. Kummer S, Hermsen D, Distelmaier F. Biotin Treatment Mimicking Graves' Disease. $N$ Engl J Med . 2016;375(7):704-706. doi:10.1056/NEJMc1602096

9. Sharma A, Baumann NA, Shah P. Biotin-Induced Biochemical Graves Disease: A Teachable Moment. JAMA Intern Med . 2017;177(4):571-572. doi:10.1001/jamainternmed.2016.9295

10. Kwok JS-S, Chan IH-S, Chan MH-M. Biotin interference on TSH and free thyroid hormone measurement. Pathology (Phila) . 2012;44(3):278-280. doi:10.1097/PAT.0b013e3283514002

11. Bowen R, Benavides R, Colón-Franco JM, et al. Best practices in mitigating the risk of biotin interference with laboratory testing. Clin Biochem . 2019;74:1-11. doi:10.1016/j.clinbiochem.2019.08.012

12. Favresse J, Burlacu M-C, Maiter D, Gruson D. Interferences With Thyroid Function Immunoassays: Clinical Implications and Detection Algorithm. Endocr Rev . 2018;39(5):830-850. doi:10.1210/er.2018-00119

13. Piketty M-L, Prie D, Sedel F, et al. High-dose biotin therapy leading to false biochemical endocrine profiles: validation of a simple method to overcome biotin interference. Clin Chem Lab Med CCLM . 2017;55(6):817-825. doi:10.1515/cclm-2016-1183

14. Laji K. Abnormal serum free thyroid hormone levels due to heparin administration. QJM . 2001;94(9):471-473. doi:10.1093/qjmed/94.9.471

15. Burch HB. Drug Effects on the Thyroid. Ingelfinger JR, ed. N Engl J Med . 2019;381(8):749-761. doi:10.1056/NEJMra1901214

16. Jaume JC, Mendel CM, Frost PH, Greenspan FS, Laughton CW. Extremely Low Doses of Heparin Release Lipase Activity into the Plasma and Can Thereby Cause Artifactual Elevations in the Serum-Free Thyroxine Concentration as Measured by Equilibrium Dialysis. Thyroid . 1996;6(2):79-83. doi:10.1089/thy.1996.6.79

17. Stevenson HP, Archbold GP, Johnston P, Young IS, Sheridan B. Misleading serum free thyroxine results during low molecular weight heparin treatment. Clin Chem . 1998;44(5):1002-1007.

18. Koulouri O, Moran C, Halsall D, Chatterjee K, Gurnell M. Pitfalls in the measurement and interpretation of thyroid function tests. Best Pract Res Clin Endocrinol Metab . 2013;27(6):745-762. doi:10.1016/j.beem.2013.10.003

19. Mendel CM, Frost PH, Cavalieri RR. Effect of free fatty acids on the concentration of free thyroxine in human serum: the role of albumin.J Clin Endocrinol Metab . 1986;63(6):1394-1399. doi:10.1210/jcem-63-61394 
20. Stockigt JR, Lim C-F. Medications that distort in vitro tests of thyroid function, with particular reference to estimates of serum free thyroxine. Best Pract Res Clin Endocrinol Metab . 2009;23(6):753-767. doi:10.1016/j.beem.2009.06.004

21. Braverman LE, Cooper D. Werner $\mathcal{E}$ Ingbar's The Thyroid: A Fundamental and Clinical Text . Lippincott Williams \& Wilkins; 2012.

22. Schussler GC. The Thyroxine-Binding Proteins. Thyroid . 2000;10(2):141-149. doi:10.1089/thy.2000.10.141

23. Knopp RH, Bergelin RO, Wahl PW, Walden CE, Chapman MB. Clinical chemistry alterations in pregnancy and oral contraceptive use. Obstet Gynecol . 1985;66(5):682-690.

24. Steingold KA, Matt DW, DeZiegler D, Sealey JE, Fratkin M, Reznikov S. Comparison of transdermal to oral estradiol administration on hormonal and hepatic parameters in women with premature ovarian failure. $J$ Clin Endocrinol Metab . 1991;73(2):275-280. doi:10.1210/jcem-73-2-275

25. Kuhl H, Jung-Hoffmann C, Weber J, Boehm BO. The effect of a biphasic desogestrel-containing oral contraceptive on carbohydrate metabolism and various hormonal parameters. Contraception . 1993;47(1):5568. doi:10.1016/0010-7824(93)90109-k

26. Geola FL, Frumar AM, Tataryn IV, et al. Biological effects of various doses of conjugated equine estrogens in postmenopausal women. J Clin Endocrinol Metab . 1980;51(3):620-625. doi:10.1210/jcem-51-3-620

27. Ben-Rafael Z, Struass JF, Arendash-Durand B, Mastroianni L, Flickinger GL. Changes in thyroid function tests and sex hormone binding globulin associated with treatment by gonadotropin. Fertil Steril . 1987;48(2):318-320. doi:10.1016/s0015-0282(16)59363-7

28. Wiegratz I, Kutschera E, Lee JH, et al. Effect of four different oral contraceptives on various sex hormones and serum-binding globulins. Contraception . 2003;67(1):25-32. doi:10.1016/S0010-7824(02)00436-5

29. Bisschop PH, Toorians AW, Endert E, Wiersinga WM, Gooren LJ, Fliers E. The effects of sexsteroid administration on the pituitary-thyroid axis in transsexuals. Eur J Endocrinol . 2006;155(1):11-16. doi:10.1530/eje.1.02192

30. Ain KB, Mori Y, Refetoff S. Reduced Clearance Rate of Thyroxine-Binding Globulin (TBG) with Increased Sialylation: A Mechanism for Estrogen-Induced Elevation of Serum TBG Concentration*. J Clin Endocrinol Metab . 1987;65(4):689-696. doi:10.1210/jcem-65-4-689

31. Glinoer D, Gershengorn MC, Dubois A, Robbins J. Stimulation of thyroxine-binding globulin synthesis by isolated rhesus monkey hepatocytes after in vivo beta-estradiol administration.Endocrinology . 1977;100(3):807-813. doi:10.1210/endo-100-3-807

32. Glinoer D, McGuire RA, Gershengorn MC, Robbins J, Berman M. Effects of estrogen on thyroxinebinding globulin metabolism in rhesus monkeys.Endocrinology . 1977;100(1):9-17. doi:10.1210/endo-100-1-9

33. Gordon D, Beastall GH, Thomson JA. The effect of tamoxifen therapy on thyroid function tests. (7):4.

34. Kostoglou-Athanassiou I, Ntalles K, Markopoulos C, Athanassiou P, Gogas J, Proukakis C. Thyroid function in postmenopausal women with breast cancer on tamoxifen. Eur J Gynaecol Oncol . 1998;19(2):150154.

35. Mamby CC, Love RR, Lee KE. Thyroid function test changes with adjuvant tamoxifen therapy in postmenopausal women with breast cancer.J Clin Oncol . 1995;13(4):854-857. doi:10.1200/JCO.1995.13.4.854

36. Anker GB, Lønning PE, Aakvaag A, Lien EA. Thyroid function in postmenopausal breast cancer patients treated with tamoxifen.Scand J Clin Lab Invest . 1998;58(2):103-107. doi:10.1080/00365519850186670 
37. Duntas LH, Mantzou E, Koutras DA. Lack of Substantial Effects of Raloxifene on ThyroxineBinding Globulin in Postmenopausal Women: Dependency on Thyroid Status. Thyroid . 2001;11(8):779-782. doi:10.1089/10507250152484637

38. Hsu SH-J, Cheng W-C, Jang M-W, Tsai K-S. Effects of Long-Term Use of Raloxifene, a Selective Estrogen Receptor Modulator, on Thyroid Function Test Profiles. Clin Chem . 2001;47(10):1865-1867. doi:10.1093/clinchem/47.10.1865

39. Ceresini G, Morganti S, Rebecchi I, et al. A one-year follow-up on the effects of raloxifene on thyroid function in postmenopausal women.Menopause $N \quad Y N$. 2004;11(2):176-179. doi:10.1097/01.gme.0000094169.64352.3b

40. Marqusee E, Braverman LE, Lawrence JE, Carroll JS, Seely EW. The effect of droloxifene and estrogen on thyroid function in postmenopausal women. J Clin Endocrinol Metab . 2000;85(11):4407-4410. doi:10.1210/jcem.85.11.6975

41. Azizi F. Thyroxine Transport and Metabolism in Methadone and Heroin Addicts. Ann Intern Med . 1974;80(2):194. doi:10.7326/0003-4819-80-2-194

42. English TN, Ruxton D, Eastman CJ. Abnormalities in thyroid function associated with chronic therapy with methadone. Clin Chem . 1988;34(11):2202-2204.

43. Bastomsky CH, Dent RR, Tolis G. Elevated serum concentrations of thyroxine-binding globulin and caeruloplasmin in methadone-maintained patients. Clin Biochem . 1977;10(3):124-126. doi:10.1016/s00099120(77)91682-4

44. Webster JB, Coupal JJ, Cushman P. Increased serum thyroxine levels in euthyroid narcotic addicts. $J$ Clin Endocrinol Metab . 1973;37(6):928-934. doi:10.1210/jcem-37-6-928

45. Gozashti MH, Mohammadzadeh E, Divsalar K, Shokoohi M. The effect of opium addiction on thyroid function tests. J Diabetes Metab Disord . 2014;13(1):5. doi:10.1186/2251-6581-13-5

46. Farhad Shahsavar, Koroush Ghanadi, Jafarzadeh M, Mehrzad Jafarzadeh, Bahram Nasiri, Shahab Forutani. Opium addiction decreases T3 uptake amount. Life Sci J . 2013;10(Special Issue 5 (Supplement 1005s)):608-610. doi:10.7537/marslsj1005s13.105

47. Yan Z, Yan H, Ou H. Human thyroxine binding globulin (TBG) promoter directs efficient and sustaining transgene expression in liver-specific pattern. Gene . 2012;506(2):289-294. doi:10.1016/j.gene.2012.07.009

48. Schussler GC, Stimmel B, Korn F. Increased serum thyroid hormone binding in narcotic addicts is due to liver disease. Am J Drug Alcohol Abuse . 1980;7(3-4):379-387. doi:10.3109/00952998008993434

49. Beex L, Ross A, Smals A, Kloppenborg P. 5-fluorouracil-induced increase of total serum thyroxine and triiodothyronine. Cancer Treat Rep . 1977;61(7):1291-1295.

50. Mitotane increases the blood levels of hormone-binding proteins. - PubMed - NCBI. Accessed April 22, 2020. https://www.ncbi.nlm.nih.gov/pubmed/1903011

51. Marshall JS, Tompkin LS. Effect of o,p'-DDD and Similar Compounds on Thyroxine Binding Globulin. J Clin Endocrinol Metab . 1968;28(3):386-392. doi:10.1210/jcem-28-3-386

52. Daffara F, De Francia S, Giuseppe Reimondo, Zaggia B,. Prospective evaluation of mitotane toxicity in adrenocortical cancer patients treated adjuvantly. Endocr Relat Cancer . 2008;15((4)):1043-1053. doi:10.1677/erc-08-0103

53. Russo M, Scollo C, Pellegriti G, et al. Mitotane treatment in patients with adrenocortical cancer causes central hypothyroidism. Clin Endocrinol (Oxf) . 2016;84(4):614-619. doi:10.1111/cen.12868

54. Bianco AC, Kim BW. Deiodinases: implications of the local control of thyroid hormone action. $J$ Clin Invest . 2006;116(10):2571-2579. doi:10.1172/JCI29812 
55. WHO cooperative trial on primary prevention of ischaemic heart disease with clofibrate to lower serum cholesterol: final mortality follow-up. Report of the Committee of Principal Investigators. Lancet Lond Engl . 1984;2(8403):600-604.

56. McKerron CG, Scott RL, Asper SP, Levy RI. Effects of clofibrate (Atromid S) on the thyroxine-binding capacity of thyroxine-binding globulin and free thyroxine. J Clin Endocrinol Metab . 1969;29(7):957-961. doi:10.1210/jcem-29-7-957

57. Barbosa J, Oliner L. Effect of clofibrate on serum thyroxine transport and free thyroxine levels. Metabolism . 1969;18(2):141-147. doi:10.1016/0026-0495(69)90108-5

58. Deyssig R, Weissel M. Ingestion of androgenic-anabolic steroids induces mild thyroidal impairment in male body builders. J Clin Endocrinol Metab . 1993;76(4):1069-1071. doi:10.1210/jcem.76.4.8473383

59. Malarkey WB, Strauss RH, Leizman DJ, Liggett M, Demers LM. Endocrine effects in female weight lifters who self-administer testosterone and anabolic steroids. Am J Obstet Gynecol . 1991;165(5 Pt 1):1385-1390. doi:10.1016/0002-9378(91)90374-z

60. Kley HK, Herrmann J, Morgner KD, Krüskemper HL. Effects of testosterone oenanthate on plasma concentrations of thyroxine, cortisol, testosterone and hormone binding proteins in patients with hypogonadism.Horm Metab Res Horm Stoffwechselforschung Horm Metab . 1973;5(4):271-274. doi:10.1055/s-00281093945

61. Small M, Beastall GH, Semple CG, Cowan RA, Forbes CD. Alteration of hormone levels in normal males given the anabolic steroid stanozolol.Clin Endocrinol (Oxf) . 1984;21(1):49-55. doi:10.1111/j.13652265.1984.tb00135.x

62. Graham RL, Gambrell RD. Changes in thyroid function tests during danazol therapy. Obstet Gynecol . 1980;55(3):395-397. doi:10.1097/00006250-198003000-00028

63. Arafah BM. Decreased levothyroxine requirement in women with hypothyroidism during androgen therapy for breast cancer. Ann Intern Med . 1994;121(4):247-251. doi:10.7326/0003-4819-121-4-199408150-00002

64. Becker U, Gluud C, Bennett P, The Copenhagen Study Group for Liver Diseases. The effect of oral testosterone on serum TBG levels in alcoholic cirrhotic men. Liver . 2008;8(4):219-224. doi:10.1111/j.16000676.1988.tb00996.x

65. Braverman LE, Ingbar SH. Effects of Norethandrolone on the Transport in Serum and Peripheral Turnover of Thyroxine. J Clin Endocrinol Metab . 1967;27(3):389-396. doi:10.1210/jcem-27-3-389

66. Tahboub R, Arafah BM. Sex steroids and the thyroid. Best Pract Res Clin Endocrinol Metab. 2009;23(6):769-780. doi:10.1016/j.beem.2009.06.005

67. Surks MI, Sievert R. Drugs and Thyroid Function. Wood AJJ, ed. N Engl J Med . 1995;333(25):1688-1694. doi:10.1056/NEJM199512213332507

68. Emerson CH, Seiler CM, Alex S, Fang SL, Mori Y, DeVito WJ. Gene expression and serum thyroxine-binding globulin are regulated by adrenal status and corticosterone in the rat. Endocrinology . 1993;133(3):1192-1196. doi:10.1210/endo.133.3.8365361

69. Oppenheimer JH, Werner SC, Martinez M. Effect of Prednisone on Thyroxine-Binding Proteins. J Clin Endocrinol Metab . 1966;26(7):715-721. doi:10.1210/jcem-26-7-715

70. Bartalena L, Martino E, Antonelli A, Pacchiarotti A, Robbins J, Pinchera A. Effect of the antileukemic agent L-asparaginase on thyroxine-binding globulin and albumin synthesis in cultured human hepatoma (HEP G2) cells. Endocrinology . 1986;119(3):1185-1188. doi:10.1210/endo-119-3-1185

71. Ferster A, Glinoér D, Vliet GV, Otten J. Thyroid Function During L-Asparaginase Therapy in Children with Acute Lymphoblastic Leukemia: Difference Between Induction and Late Intensification. J Pediatr 
Hematol Oncol . 1992;14(3):192-196.

72. Heidemann PH, Stubbe P, Beck W. Transient secondary hypothyroidism and thyroxine binding globulin deficiency in leukemic children during polychemotherapy: An effect of L-asparaginase. Eur J Pediatr . 1981;136(3):291-295. doi:10.1007/BF00442997

73. Garnick MB, Larsen PR. Acute Deficiency of Thyroxine-Binding Globulin during L-Asparaginase Therapy. $N$ Engl J Med . 1979;301(5):252-253. doi:10.1056/NEJM197908023010506

74. Fadilah SA, Faridah I, Cheong SK. Transient hyperthyroidism following L-asparaginase therapy for acute lymphoblastic leukemia.Med J Malaysia . 2000;55(4):513-515.

75. Shakir KM, Kroll S, Aprill BS, Drake AJ, Eisold JF. Nicotinic acid decreases serum thyroid hormone levels while maintaining a euthyroid state. Mayo Clin Proc . 1995;70(6):556-558. doi:10.4065/70.6.556

76. O'Brien T, Silverberg JD, Nguyen TT. Nicotinic acid-induced toxicity associated with cytopenia and decreased levels of thyroxine-binding globulin. Mayo Clin Proc . 1992;67(5):465-468. doi:10.1016/s00256196(12)60393-0

77. Drinka PJ. Alterations in thyroid and hepatic function tests associated with preparations of sustainedrelease niacin. Mayo Clin Proc . 1992;67(12):1206. doi:10.1016/s0025-6196(12)61154-9

78. Cashin-Hemphill L, Spencer CA, Nicoloff JT, et al. Alterations in serum thyroid hormonal indices with colestipol-niacin therapy. Ann Intern Med . 1987;107(3):324-329. doi:10.7326/0003-4819-107-2-324

79. Wang R, Nelson JC, Wilcox RB. Salsalate and Salicylate Binding to and their Displacement of Thyroxine from Thyroxine-Binding Globulin, Transthyretin, and Albumin. Thyroid . 1999;9(4):359-364. doi:10.1089/thy.1999.9.359

80. Larsen PR. Salicylate-induced increases in free triiodothyronine in human serum. Evidence of inhibition of triiodothyronine binding to thyroxine-binding globulin and thyroxine-binding prealbumin. $J$ Clin Invest . 1972;51(5):1125-1134. doi:10.1172/JCI106905

81. Faber J, Waetjen I, Siersbaek-Nielsen K. Free thyroxine measured in undiluted serum by dialysis and ultrafiltration: effects of non-thyroidal illness, and an acute load of salicylate or heparin. Clin Chim Acta Int J Clin Chem . 1993;223(1-2):159-167. doi:10.1016/0009-8981(93)90072-c

82. McConnell RJ. Abnormal thyroid function test results in patients taking salsalate. JAMA . 1992;267(9):1242-1243.

83. Samuels MH, Pillote K, Asher D, Nelson JC. Variable Effects of Nonsteroidal Antiinflammatory Agents on Thyroid Test Results. J Clin Endocrinol Metab . 2003;88(12):5710-5716. doi:10.1210/jc.2002-021869

84. Stockigt JR, Lim CF, Barlow JW, et al. Interaction of furosemide with serum thyroxine-binding sites: in vivo and in vitro studies and comparison with other inhibitors. J Clin Endocrinol Metab . 1985;60(5):10251031. doi:10.1210/jcem-60-5-1025

85. Newnham HH, Hamblin PS, Long F, Lim C-F, Topliss DJ, Stockigt JR. Effect of oral frusemide on diagnostic indices of thyroid function. Clin Endocrinol (Oxf) . 1987;26(4):423-431. doi:10.1111/j.13652265.1987.tb00799.x

86. Zhang Y-X, Shen C-H, Lai Q-L, et al. Effects of antiepileptic drug on thyroid hormones in patients with epilepsy: A meta-analysis.Seizure . 2016;35:72-79. doi:10.1016/j.seizure.2016.01.010

87. Surks MI, DeFesi CR. Normal serum free thyroid hormone concentrations in patients treated with phenytoin or carbamazepine. A paradox resolved. JAMA . 1996;275(19):1495-1498.

88. Chopra IJ, Van Herle AJ, Teco GN, Nguyen AH. Serum free thyroxine in thyroidal and nonthyroidal illnesses: a comparison of measurements by radioimmunoassay, equilibrium dialysis, and free thyroxine index. $J$ Clin Endocrinol Metab . 1980;51(1):135-143. doi:10.1210/jcem-51-1-135 


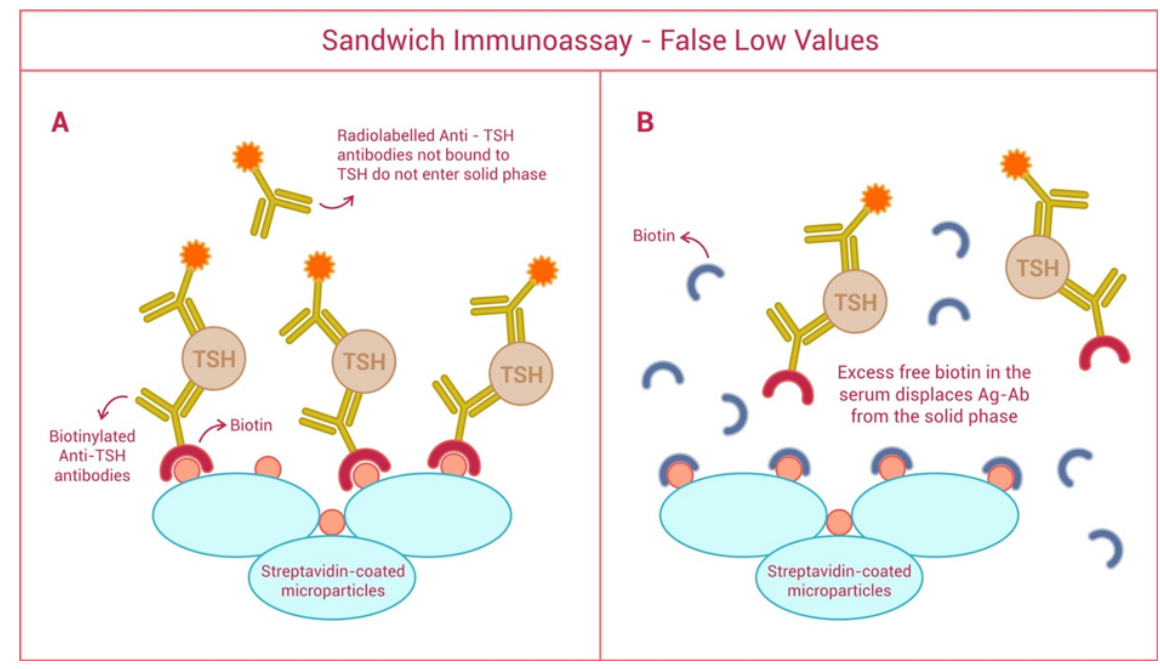

Competitive Immunoassay - False High Values

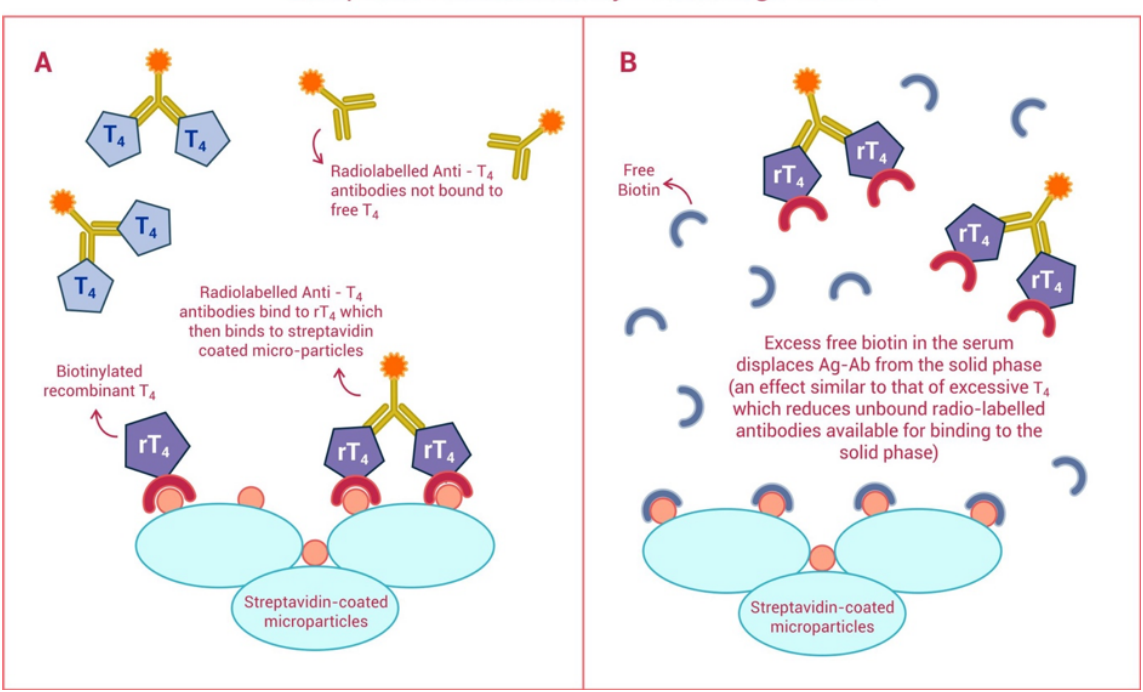

